Aus dem Evangelischen Krankenhause (Eduard Morian-Stiftung) und der Spezialabteilung für verkrüppelte Krieger im Reservelazarett Hamborn a. Rh.

\title{
Die wirtschaftliche Bedeutung der operativen Knochenverlängerung und Gelenkmobilisation.
}

Von Dr. Emil Schepelmann, Leitender Arzt der Abteilung, Fachärztlicher Beirat für Chirurgie im VII. Armeekorps.

Die großen Fortschritte der Chirurgie und die gründliche Vorbildung der praktischen Aerzte haben trotz der verheerenden Wirkung der modernen Kampfmittel gegenüber den großen Kriegen des vorigen Jahrhunderts eine erhebliche Verminderung der relativen Mortalitätsund Amputationsziffer gebracht; es sind eine große Zahl Schwerstverwundeter am Leben erhalten und konservativ behandelt, die früher zugrundegegangen oder zum mindesten amputiert worden wären. Dieses konserva tive Verfahren läßt schon ohne weiteres darauf schließen, daB bei der Schwere vieler Knochen- und Gclenkverletzungen die Rücksicht auf das Leben der Patienten im Vordergrunde des Interesses stand, hinter dem die Sorge um anatomisch und funktionell gün. stige Heilung zurücktreten mußte. Wir sehen daher eine große Zahl Verwundeter zur Entlassung aus dem Heere kommen, deren Gelenke versteift, deren Beine hochgradig, manchmal bis zur Marschunfähigkeit verkürzt sind.

Während nun die Fürsorge für die Amputierten außerordentlich weitgehend ist und die hervorragendsten Chirurgen und die besten Lazarette mit der Behandlung der Amputierten betraut sind, werden unsere Krieger mit knöchern versteiften Gelenken und mit hochoradig verkürzten Oberschenkeln im állgemeinen nach Heilung der Wunden und Verordnung eines hohen Schuhes als D.U. entlassen, obwohl gerade sie in vielen Fällen die beste Aussicht für chirurgische Behandlung darböten. Ist es doch schon oft gelungen ${ }^{1}$ ), schwer verkrüppelten Kriegern ihre Gelenke operativ zu mobilisieren oder ihre verkürzten Beine auf normale oder nahezu normale Länge zu bringen. Zu den l. c. bereits publizierten Fällen kommen noch zahlreiche andere hinzu, die später in Behandlung traten und die dank der wachsenden Erfahrung und Technik und der zunehmenden Schulung des Persopals zu noch besseren Erfolgen gebracht wurden.

Wenn der Staat den Amputierten seine allergrößte Fürsorge zuteil werden läßt, so ist das ein Akt der Humanität, Dankbarkeit und Pflicht gegen die tapferen Verwundeten; ein nennenswerter finanzieller Gewinn - von dem volkswirtschaftlichen soll zunächst nicht die Rede sein - erwächst ihm - bisher wenigsterıs - nicht daraus, weil die Rente ohne Rücksicht auf die Brauchbarkeit der Prothese - glatten Verlust vorausgesetzt - festgelegt ist und für jede einzelne Amputation eine bestimmte Höhe hat, die auch durch die Gewährung einer Prothese vielfach nicht geändert wird.

Anders liegen die Verhältnisse bei denjenigen schweren Verwundungen, deren Behandlung ich mir von Kriegsbeginn an zur besonderen Aufgabe gemacht habe: den Gelenkversteif ungen und den hochgradigen Beinverkürzungen.

Schon fibröse Versteifungen von Gelenken bilden ein dankbares Gebiet für Spezialbehandlung, da durch geschultes Personal, durch interessierte Aerzte und durch eigens konstruierte Apparate ${ }^{2}$ ) selbst dort noch eine Beseitigung der Kontrakturen möglich ist, wo andere Behandlungen versagten.

Aber auch knöcherne Gelenkankylosen sind einer Therapie zugängig; unter wesentlicher Vereinfachung älterer Methoden habe ich mit einem Operations- und Nachbehandlungsverfahren ${ }^{3}$ ) in zahlreichen, bisher nur zum Teil veröffentlichten Fällen eine Mobilisierung von Ankylosen vorgenommen und Aussicht gewonnen, nach genügender Anpassungsfrist von etwa zwei bis drei Jahren die Erwerbsfähigkeit der so Qperierten um 10\% oder mehr zu erhöhen. Es sind unter anderem Ellbogen- und Kniegelenke operiert, die nach völliger Versteifung in ungünstiger Stellung durch die Operation fast normal beweglich gemacht wurden (Beitr. z. klin. Ghir. 108, H. 5, Fig. 501, 56, 57, $58,77)$ und demnach begründete Aussicht auf Wiedererlangung der vollen Erwerbsfähigkeit darbieten. Stellte sich doch kürzlich ein Kriegsbeschädig ter (Fig. 56, l. c.) mit operativ mobilisiertem rechten Ellbogen vor, der bereits anderthalb Jahre nach der Operation als vollwertiger Kohlenhauer $12 \mathrm{M}$ pro Tag verdiente. Spätere

1) E. Schepelmann, M. m. W. 1917 Nr. 11; Beitr. z. klin. Chir. 108. 1918 H. 5 und 109, H. 4; D. Zschr. f. Chir. 1918

2) E. Sche pelmann, M. m. W. 1916 Nr. 3, 43 u. 1917 Nr. 7.

3) Beitr. z. klin. Chir. 108. 1918 H. 5. 
Nachun tersuchungen und Mitteilungen in der Fachpresse sollen noch Aufschluß über den Enderfolg geben; einstweilen will ich nur mit einer Erhöhung der Erwerbsfähigkeit um 10\% rechnen; lege ich $20 \mathrm{Jahre}$ als Alter des operierten Soldaten zugrunde, so würde eine Rentenminderung um $10 \%$ dem Staate einen jährlichen Gewinn von $54 \mathrm{M}$ (bei Gemeinen) bringen, was bei Hinzurechnung von Zinsen und Zinseszinsen in den wahrscheinlich noch zu erlebenden 45 Jahren nach der Formel

$$
\mathrm{y}_{\mathrm{n}}=\mathrm{c}\left(1+\frac{100}{\mathrm{p}}\right) \cdot\left[\left(1+\frac{\mathrm{p}}{100}\right)^{\mathrm{n}}-1\right]^{1}
$$

« dem Schlußwert 9055,67 $\mathrm{M}$ führen müßte.

Noch mehr fallen die hohen Renten in die Augen, wenn man die Verwundeten mit hochgradigen Beinverkürzungen in Betracht zieht. Lasse ich in meiner Statistik der oben zitierten Arbeit „Ueber die operative Verlängerung stark verkürzter Gliedmaßen“ den Fall 19 , ein $2^{1} / 2$ jähriges Kind, au ßer acht, so ergeben die 18 restierenden operativ behandel ten Fälle eine Gesamtverkürzung von $118 \mathrm{~cm}$ oder pro Patient von 6,56 cm, der nach Abschluß des Heilverfahrens noch eine Verkürzung von insgesamt $21^{1} / \mathrm{s} \mathrm{cm}=1,14 \mathrm{~cm}$ pro Patient gegenüberstand, sodaß die Verlängerung pro Kopf $5,42 \mathrm{~cm}$ betrug! Es ist sicher nicht zu weit gegangen, wenn ich bei einer derartigen Verkürzung von $6,56 \mathrm{~cm}$ eine dauernde, lebenslängliche Erwerbseinbuße von $331 / 3 \%$, bei der nach der Operation noch restierenden Verkürzung um 1,14 cim jedoch eine volle Erwerbsfähigkeit annehme. Der Zuwachs an Erwerbsfähigkeit beträgt demnach $33^{1 / 3} \%$. Bei dem Durchschnittsalter der behandelten Patienten ( $24 \mathrm{Jahre}$ ) ist mit einer Lebensaussicht von 40 Jahren zu rechnen, sodaß 40 Jahre hindurch jedem einzelnen (ich nehme an, es hätte sich nur um Verwundrte (Gemeine) gehandelt) jährlich $180 \mathrm{M}$ Rente zu zahlen sein würden; durch die Operation eriibrig te sich die Rentenzahlung, und der Staat gewänne dadurch bei Benutzung der oben zitierten Formel in jedem einzelnen Falle $22831,20 \mathrm{M}$, zu der noch Tie Verstümmlungszulage treten würde, die in jedem Falle von mehr als $5 \mathrm{~cm}$ Verkürzung in Betracht kommen könnte ${ }^{2}$ ). Sie beträgt für den gemeinen Mann monatlich $27 \mathrm{M}$, also jährlich $324 \mathrm{M}$, und würde gegebenenfalls in den 18 Fällen $16 \mathrm{ma}$ zum Fortfall gebracht sein. Diese 16 Fälle wiesen ein Gesamtalter von 369 Jahren, also pro Person ein Durchschnittsalter von 23,06 Jahren auf, soda $B$ auch hier mit einer Lebensaussicht von 40 Jahren zu rechnen wäre. Dann berechnet sich der Endwert dieser 40jährigen Rentenzahlung aut $47900,16 \mathrm{M}$, was zu sammen mit der oben genannten $331 / 3 \%$ igen Rente von 22831,20 M 70 731,36 M ergeben würde.

Schon durch den Fortfall der Rente und der Verstümmelungszulage infolge der Operation erzielte also der Staat gewissermaßen einen Gewinn von fast $71000 \mathrm{M}$ pro Person; in Wirklichkeit ist der Vorteil für ihn noch weit größer, da der Kriegsbeschädig te nunmehr wieder vollständig erwerbsfähig geworden ist und dem Staate seine Arbeitskraft und seine Steuern zugutekommen lassen kann. Auch der Kriegsbeschädigte selbst hat durch die Operation einen erheblich größeren wirtschaftlichen Gewinn erreicht, da die Rente ihm doch nur einen kleinen Teil seiner verlorenen Erwerbsfähigkeit ersetzt, während er mit nunmehr normalem Körperbaı und erhöhter Leistungsfähigkeit ganz andere Einnahmen zu erzielen vermag.

Der kosmetische Erfolg geht aus den Fig. 5, 7, 8, 9, 12, 13, 14, 15, 16, 18, 19 meiner zitierten Arbeit in den Beitr. z. k]in. Chir. 109, H. 4 hervor.

1) Hierbei bedeutet y den Endwert, $n$ die Anzahl der Jahre, c die jährlich : Rente und $\mathrm{p}$ den Prozentsatz.

$\left.{ }^{2}\right)$ In der ,Dienstanweisung zur Beurteilung der Militärdienstfähigkeit" heißt es in Ziffer 139: Für die Brurte: lung einer etwaigen Verstümmelung an den unteren Gliedmaßen ist immer ausschlagg"bend, ob und in welchem Grade der Fuß gebrauchsfähig geblieben ist; Störungen in der Gebrauchsfähigkeit der Ober- und Unterschenkel sind dem Verlust des Fußes dann gleich zu erachten, wenn durch ein falsches Gelenk (Pseudarthrose) des Oberschenkels, starke Winkelstellurg im Knie, hochgradige Spitzfußstellıng und ähnliche Zustände der Fuß zum Gehen unbrauchbar geworden ist. Verkürzung der Untergliedmaßen bis zu $5 \mathrm{~cm}$, in Strackstellung gemessen, genügt zur Erfüllung des Begriffes der Verstümmelung im allgemeinen noch nicht, da eine derartige Verkürzung noch durch Beckenrerschiebung und einen um etwa $1,5 \mathrm{~cm}$ erböhten Absatz usw. genügend ausgeglichen zu werden pflegt. Wird das Gehen hierdurch nicht ermöglicht und handelt es sich um a u s g a dehn te Verkürzungen, so ist die Annahme einer Verstümmelung zulässig.“ §140: ..Bei der Beurteilung, ob eine Verstümmeluug vorliegt, bleibt die durch etwaige künstliche Hilfe (mit Ausnahme der unter Ziffer 139 erwähnten hohen. Absätze usw.) erzielte Besserung außer Betracht. Die Gebrauchs- und Leistungsfähigkeit des Gliedes für die Beurteilung, ob Verstïmmelung vorliegt (im Gegensatz zur Beurteilung des Grades der Erwerbsunfähigkeit, Ziffer $123 \mathrm{Abs}$. 2), ist so zu bewerten, wie sie sich darstellt, wenn der Betreffende auf die Benutzung des Gliedes ohne Hilfs mittel angewiesen wäre. Die Benutzung eines Stockes beim Gehen rechnet nicht ohne weiteres zum Gebrauch künstlicher Hilfsmittel."“
Nicht unerwähnt möchte ich hier den Umstand lassen, daß durch die schlechte Heilung der Oberschenkelfraktur aụßer einer Verkürzung des Beines auch eine. mit der Beckensenkung zusammenhängende schwere Deformität des ganzen Rumpfes, namentlich seines Skelettes, entsteht.

Von den von mir operierten Patienten, gelangten einige durch Zufall ins Krankenhaus, andere wurden mir durch Aerzte, Sanitäts offiziere oder Offiziere zugeführt oder suchten mich durch Empfehlung anderer Operierter spontan nach ihrer Entlassung als D.U. auf. Wenn ich jedoch in großen Städten die Zahl schwer hinkender Kriegsbeschädigter betrachte, wenn ich an die Statistik Zahradnickys ${ }^{1}$ ) erinnere, nach der von 100 Gelenkverwundungen 12,9 , oder gar an die Statistik Könneckes 2), nach der mehr als $50 \%$ von Gelenk. verwundungen zur Ankylose führen, so ist das von mir bearbeitete Material nur ein unscheinbarer Teil des Materials, das sich in der näheren Umgebung meines Wohnortes an Verkrüppelten vorfindet. Eine nicht geringe Anzahl von ihnen würde sich gewiß gern einer Operation unterziehen, wenn sie nur die Möglichkeit ahnten. daß ihnen geholfen werden könnte, oder wenn sie im Krankenhause die Erfolge der opera. tiven Therapie persönliuh an Beispielen kennen lemten.

Es würde sich deshalb empfehlen, in jedem Korpsbezirk die Nachuntersuchungen von Kriegsrentenempfängern mit knöchernen Versteifungen der großen Gelenke der Gliedmaßen oder mit Beinverkürzungen zweiten und dritten Grades von Aerzten vornehmen zu lassen, die sich spezialistisch mit der Behandlung derartiger Verkrüppelungen befaßt haben. Sie könnten die Kriegsbeschädigten auf die Möglichkeit einer Heilung aufmerksam machen und eventuell einem einschlägigen Lazarett zuführen. 